\title{
Antimicrobial resistance profiles of common mastitis pathogens on Canadian dairy farms
}

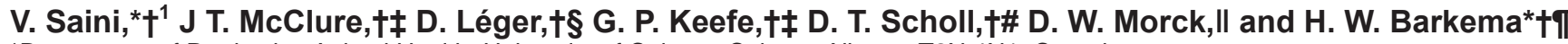 \\ *Department of Production Animal Health, University of Calgary, Calgary, Alberta, T2N 4N1, Canada \\ †Canadian Bovine Mastitis Research Network, C. P. 5000, St-Hyacinthe, Québec, J2S 7C6, Canada \\ ‡Department of Health Management, Atlantic Veterinary College, University of Prince Edward Island, Charlottetown, Prince Edward Island, \\ C1A 4P3, Canada \\ §Public Health Agency of Canada, Laboratory for Foodborne Zoonoses, Guelph, Ontario, N1G 5B2, Canada \\ \#South Dakota Agricultural Experiment Station, South Dakota State University, Brookings SD, 57007 \\ IIDepartment of Biological Sciences, and Comparative Biology and Experimental Medicine, University of Calgary, Calgary, Alberta, \\ T2N 4N1, Canada \\ IDepartment of Reproduction, Obstetrics and Herd Health, Ghent University, Merelbeke, Belgium
}

\section{ABSTRACT}

Monitoring of antimicrobial resistance (AMR) in bacteria has clinical and public health significance. The present study determined prevalence of AMR in common mastitis pathogens Staphylococcus aureus, including methicillin-resistant Staph. aureus (MRSA; n = 1,810), Escherichia coli $(\mathrm{n}=394)$, and Klebsiella species $(\mathrm{n}=139)$, including extended-spectrum $\beta$-lactamase (ESBL)-producing E. coli and Klebsiella species, isolated from milk samples on 89 dairy farms in 6 Canadian provinces. Minimum inhibitory concentrations (MIC) were determined using the Sensititer bovine mastitis plate (Trek Diagnostic Systems Inc., Cleveland, $\mathrm{OH}$ ) and a National Antimicrobial Resistance Monitoring System gram-negative panel containing antimicrobials commonly used for mastitis treatment and control. Denim blue chromogenic agar and real-time PCR were used to screen and confirm MRSA, respectively. Resistance proportion estimates ranged from $0 \%$ for cephalothin and oxacillin to $8.8 \%$ for penicillin in Staph. aureus isolates, and $15 \%$ of the resistant Staph. aureus isolates were multidrug resistant. One MRSA isolate was confirmed (prevalence: $0.05 \%$ ). Resistance proportion estimates ranged from $0 \%$ for ceftriaxone and ciprofloxacin to $14.8 \%$ for tetracycline in E. coli, and $0 \%$ for amikacin, ceftiofur, ciprofloxacin, and nalidixic acid to $18.6 \%$ for tetracycline in Klebsiella species isolates. Further, 62.8 and $55 \%$ of the resistant E. coli and Klebsiella species isolates were multidrug resistant, respectively. Resistance to $>5$ and $>2$ antimicrobials was most common in E. coli and Klebsiella species isolates, respectively, and no ESBL producers were found. Prevalence of AMR in bovine mastitis pathogens was

Received January 23, 2012.

Accepted April 2, 2012.

${ }^{1}$ Corresponding author: vsaini@ucalgary.ca low. Most gram-negative udder pathogens were multidrug resistant; MRSA was rarely found, and ESBL E. coli and Klebsiella species isolates were absent in Canadian milk samples.

Key words: Escherichia coli, Klebsiella, methicillinresistant Staphylococcus aureus, extended-spectrum $\beta$-lactamase

\section{INTRODUCTION}

In the context of food-animal production systems, mastitis is the most common and most economically significant disease affecting dairy cattle. It is the leading cause of antimicrobial use on dairy farms (Saini et al., 2012). A variety of bacteria can be isolated from bovine mastitis cases. Staphylococcus aureus and Escherichia coli are the most common causes of contagious and environmental clinical mastitis, respectively (Barkema et al., 1998), whereas Klebsiella is an emerging pathogen with rising incidence in North America (Zadoks and Muñoz, 2007).

Antimicrobial therapy is commonly implemented for mastitis prevention and control. Unfortunately, despite the best possible antimicrobial treatments, failures of bacteriological cure are common, especially for Staph. aureus mastitis, and antimicrobial resistance (AMR) is considered one of the reasons for low cure rates (Barkema et al., 2006). Additionally, AMR in bacteria is a public health hazard, and antimicrobial use is considered a potentially important driver of AMR. For example, cloxacillin, an antimicrobial similar to methicillin/oxacillin, is extensively used for dry cow therapy (Saini et al., 2012), and it is hypothesized that cloxacillin use may select for methicillin-resistant Staph. aureus (MRSA) isolates. Methicillin-resistant Staph. aureus have been isolated from mastitis milk samples and have the potential to complicate treatment of bovine mastitis (Vanderhaeghen et al., 2010). Further, live- 
stock can be reservoirs of resistant genes such as those associated with the production of extended-spectrum $\beta$-lactamases (ESBL) in Enterobacteriaceae that could be transferred to humans (Fey et al., 2000). In addition to expanded-spectrum cephalosporins (e.g., ceftriaxone, ceftazidime, cefotaxime, and ceftiofur), ESBL producers frequently carry resistance determinants that confer resistance to fluoroquinolones, aminoglycosides, and trimethoprim (TMP)-sulfamethoxazole combinations. Ceftiofur is commonly used to treat dairy cattle, including systemic treatment of coliform mastitis (Wagner and Erskine, 2006), and ESBL E. coli and Klebsiella species have been isolated in milk from dairy cattle (Hammad et al., 2008; MARAN-2008, 2009) and from milk filters on a dairy farm (Dolejska et al., 2011). In general, the emergence and transfer of AMR bacteria or genetic determinants from animals to human populations via food chain is a growing concern (Piddock, 1996). In the context of dairy farming, even though most milk produced is pasteurized, unpasteurized milk is still consumed by dairy producers and their families, and raw milk cheese can be sold to consumers; disease outbreaks have been linked to the consumers of raw milk (Oliver et al., 2005). It is therefore important to determine AMR in food-animal pathogens at the pre- and postharvest stages of food production and to monitor AMR profile patterns in them over years for food safety, animal health, and public health reasons.

The leading body in Canada for data collection on AMR in bacterial isolates from chicken, swine, and beef cattle meat is the Canadian Integrated Program for Antimicrobial Resistance Surveillance. Unfortunately, it does not collect information on AMR profiles of common mastitis pathogens at the retail or farm level. Information is lacking on the prevalence of AMR in bovine mastitis pathogens in milk in Canada. Some of the previous studies conducted to determine AMR profiles of bovine mastitis pathogens in Canada included isolates from clinical mastitis cases submitted to diagnostic laboratories; a disk diffusion test involving different antimicrobials than being used presently was commonly employed in these studies (MacDonald et al., 1973; Hariharan et al., 1974). None of these studies collected information on the prevalence of MRSA and ESBL E. coli and Klebsiella species in milk samples and did not collect isolates from subclinical mastitis cases or IMI. Therefore, epidemiological differences in the sources of isolates could result in a biased assessment of AMR in bovine mastitis pathogens in Canada.

The objectives of the present study were to determine (1) antimicrobial resistance profile patterns of Staph. aureus, E. coli, and Klebsiella species pathogens isolated from subclinical and clinical mastitis cases and IMI, and (2) prevalence of MRSA and ESBL E. coli and Klebsiella species in milk samples from Canadian dairy herds.

\section{MATERIALS AND METHODS}

\section{Dairy Cattle Herd Selection}

Milk samples were collected from the National Cohort of Dairy Farms of the Canadian Bovine Mastitis Research Network (CBMRN), which consisted of 91 commercial dairy farms located in 4 regions across Canada [Alberta, Ontario, Québec, and the Maritime Provinces (Prince Edward Island, Nova Scotia, and New Brunswick)]. Herd selection criteria for the present study were described by Reyher et al. (2011). In short, dairy herds were selected to replicate the provincial proportion of freestall systems to within 15 percentage points and to be uniformly distributed among 3 strata of the most recent 12-mo bulk tank SCC $(\leq 150,000$ cells $/ \mathrm{mL},>150,000$ and $\leq 300,000$ cells $/ \mathrm{mL},>300,000$ cells $/ \mathrm{mL}$ ); herds with a 3-times-daily milking schedule and herds with $<80 \%$ (or < $<15$ ) Holstein-Friesian lactating and dry cows at the time of enrollment were excluded. Further, eligible dairy herds must have been participating in a Dairy Herd Improvement data collection program. Eligible dairy farms were identified and contacted by regional center coordinators. Written consent to participate in the research cohort was obtained. Two dairy herds elected not to participate at initiation of the study. In each coordinating center, an investigator and technicians were responsible for the data collection activities related to farms located in that center's area.

\section{Sampling and Bacterial Culturing}

Different sets of milk samples were collected (Reyher et al., 2011) from (1) cows with clinical mastitis (M1 to M3), (2) nonclinical lactating cows (L1 to L7), and (3) cows before dry-off (DC1 and DC2) and after calving (FC1 and FC2). All producer-diagnosed clinical mastitis cases were sampled (M1) and then resampled twice at 2-wk intervals (M2 and M3). The M1 samples were taken before antimicrobial treatment, whereas the M2 and M3 samples were taken after antimicrobial treatment.

The second set of milk samples was collected from nonclinical lactating cows; a subsample of 15 fresh and lactating cows was selected randomly per farm using a computer-driven method of random sampling without replacement. They were aseptically sampled and resampled once every $3 \mathrm{wk}$ for a total of 3 samplings in the winter of 2007 (L1, L2, and L3); another subsample of 15 lactating cows was sampled once a week for $7 \mathrm{wk}$ in 
the summer of 2007 (L1 to L7). The 2008 data collection was similar to that of 2007 except that both summer and winter samplings were done 3 times at 3 -wk intervals and that the fresh cows were not included in the lactating cow subsample.

The third set of milk samples was collected from another selected group of 15 cows that were expected to remain in the herd until at least 2 wk after calving. A subsample was aseptically sampled before dry-off (DC1 and DC2) and after calving (FC1 and FC2) in 2007 and this continued in 2008 as well. Samples were frozen at $-20^{\circ} \mathrm{C}$ and shipped to the regional CBMRN laboratory where bacterial culturing and identification of the milk samples was done according to National Mastitis Council guidelines (Hogan et al., 1999). In short, Staph. aureus isolates were identified as gram-positive cocci by Gram stain, a positive catalase test (Fisher Scientific Company, Nepean, ON, Canada), double-zone hemolysis on Columbia agar (Oxoid Ltd., Basingstoke, UK) with $5 \%$ sheep blood (Alere Inc., Ottawa, ON, Canada), a positive tube coagulase test, and a positive DNase test (Becton Dickinson, Sparks, MD). Escherichia coli and Klebsiella species isolates were identified as gramnegative rods by Gram stain, lactose fermenters on MacConkey agar (Neogen Corp., Lansing, MI), and a negative oxidase test (Becton Dickinson). In addition, a triple-sugar iron agar slant, a urea, a citrate, and a spot-indole test were also set up for identifying gramnegative pathogens (Becton Dickinson; Oxoid Ltd.). Isolates that were motile, positive for indole production, negative for urease production and citrate utilization, produced acid on the slant, and acid and gas in the butt of a triple-sugar iron agar slant were identified as E. coli. Klebsiella species isolates were nonmotile, negative for indole production, produced urease, and utilized citrate. Isolates of growth from all culture-positive quarters that were considered significant according to National Mastitis Council guidelines were conserved in the isolate repository at the University of Montreal (National Cohort of Dairy Farms, St-Hyacinthe, Québec; see NCDF, 2009 for details on conservation).

Because multiple isolates could be coming from a single cow, we decided to randomly include only one isolate per quarter using the computer-driven randomization process of sampling without replacement. Selected isolates were shipped from the isolate repository at the University of Montreal to the Atlantic Veterinary College at the University of Prince Edward Island for AMR determination. Clinical mastitis was defined as an inflammation of the udder leading to occurrence of flakes, clots, or other gross alterations in milk, whereas subclinical mastitis was defined as SCC >200,000 cells/ $\mathrm{mL}$ from a cow without clinical signs of mastitis. Intramammary infection was defined as a culture-positive sample with a minimum number of colonies cultured per $0.01 \mathrm{~mL}$ of milk $\geq 1$ and without reference to SCC (Dohoo et al., 2011; Reyher et al., 2011). The term IMI referred specifically to the presence of an infectious organism in the udder without any reference to SCC (Berry and Meaney, 2006).

\section{Antimicrobials}

The MIC of these isolates were determined using the Sensititer microdilution system (Trek Diagnostic Systems Inc., Cleveland, OH). Sensititer Mastitis plate format CMV1AMAF, and Sensititer National Antimicrobial Resistance Monitoring System (NARMS) gram-negative plate format CMV1AGNF were used for gram-positive and gram-negative organisms, respectively. The bovine mastitis plate contained antimicrobials that are commonly used for mastitis prevention and control, including ampicillin, ceftiofur, cephalothin, erythromycin, oxacillin, penicillin, penicillin-novobiocin combination, pirlimycin, sulfadimethoxine, and tetracycline (Table 1). The NARMS gram-negative plate included the following antimicrobials: amikacin, ampicillin, amoxicillin-clavulanic acid (CLA) combination, ceftriaxone, chloramphenicol, ciprofloxacin, TMPsulfamethoxazole combination, cefoxitin, gentamicin, kanamycin, nalidixic acid, sulfisoxazole, streptomycin, tetracycline, and ceftiofur (Table 2).

\section{Sensititer System MIC Method}

Antimicrobial susceptibility testing was done as per manufacturer's instructions (Trek Diagnostic Systems Inc.). The MIC values of various antimicrobial-isolate combinations were determined using the Sensititer Auto Reader. In case of sulfadimethoxine, the MIC results were determined manually, as advised by Trek Diagnostics. Thereafter, $10 \mu \mathrm{L}$ of Sensititer MuellerHinton broth inoculated with Staph. aureus suspension was pipetted onto denim blue agar plates (Chromogenic MRSA Screening Agar, Oxoid, Nepean, ON, Canada), and streaked for individual colonies for detection of methicillin resistance. Concurrently, a plate of Sensititer Mueller-Hinton agar containing $0.25 \mu \mathrm{g} / \mathrm{mL}$ of penicillin $\mathrm{G}$ was also inoculated for detecting penicillin resistance. Denim blue agar plates and Mueller-Hinton agar plates containing $0.25 \mu \mathrm{g} / \mathrm{mL}$ of penicillin $\mathrm{G}$ were incubated at $37 \pm 2^{\circ} \mathrm{C}$ for 18 and $48 \mathrm{~h}$, respectively. Discrete denim blue colonies against a white background were presumptive positive for MRSA. An MRSA-positive isolate was confirmed for the presence of penicillin binding protein $\mathrm{PBP} 2^{\prime}$ using a $\mathrm{PBP} 2^{\prime}$ latex agglutination test (Oxoid). Subsequently, genotypic confirmation of the presence of mecA gene for methicil- 


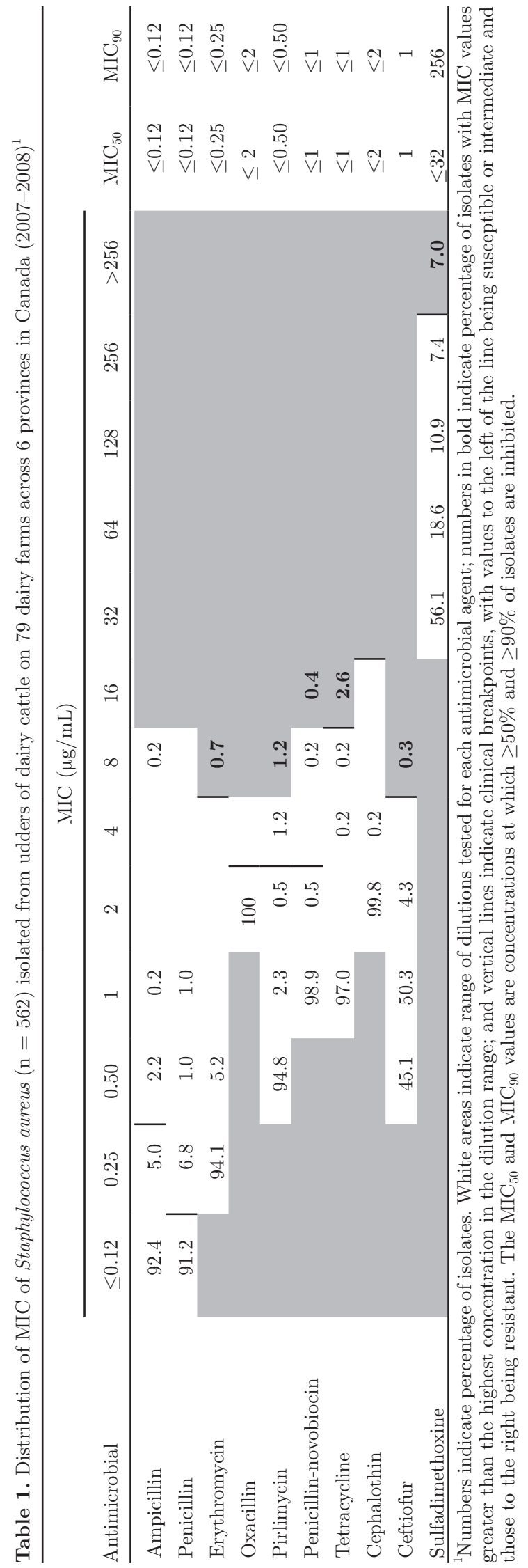

lin resistance was done using a real-time PCR assay (Paule et al., 2005). Growth on Mueller-Hinton agar plates containing $0.25 \mu \mathrm{g} / \mathrm{mL}$ of penicillin $\mathrm{G}$ indicated penicillin resistance in Staph. aureus isolates. Escherichia coli and Klebsiella species isolates were screened for ESBL production based on ceftriaxone MIC of $\geq 2$ $\mu \mathrm{g} / \mathrm{mL}$ as recommended by the Clinical and Laboratory Standards Institute (CLSI, 2008). Later, a Sensititer ESBL confirm plate was used on suspect isolates for confirmation of ESBL production, and all ESBL isolates were to be confirmed as E. coli or Klebsiella species using a Sensititer gram-negative identification plate. The following reference strains were included in the study: Staph. aureus ATCC 25923, Staph. aureus ATCC 29213, E. coli ATCC 25922, and Klebsiella pneumoniae ATCC 700603. Isolates of interest in this study (i.e., Staph. aureus, E. coli, and Klebsiella species) were stored in skim milk in a commercial freezer at $-20^{\circ} \mathrm{C}$.

\section{Statistical Analyses}

Data were checked for unlikely observations; however, no unlikely values were excluded. The MIC values were determined and MIC was defined as the lowest concentration of an antimicrobial that inhibited any visible growth of an isolate. For the antimicrobial combinations such as penicillin-novobiocin, amoxicillin-CLA, and TMP-sulfamethoxazole, the MIC of the first agent (penicillin, amoxicillin, or TMP) was reported as the MIC for the combination. We also determined $\mathrm{MIC}_{50}$ and $\mathrm{MIC}_{90}$ values for various isolate-antimicrobial combinations. The $\mathrm{MIC}_{50}$ and $\mathrm{MIC}_{90}$ value represented the minimum concentration of an antimicrobial that inhibited the visible growth of $\geq 50 \%$ and $\geq 90 \%$ of the microorganisms, respectively. Thereafter, the isolates were categorized as susceptible, intermediate, or resistant on the basis of CLSI- and NARMS-based MIC breakpoints (CLSI 2000a,b, 2008; NARMS, 2011). Intermediate isolates were combined with resistant isolates for the purpose of statistical analyses. Proportions of isolates that were resistant to at least one antimicrobial were determined, and 95\% exact binomial confidence intervals were calculated. Proportions of multidrug resistant (MDR) isolates were also determined. Multidrug resistance was defined as resistance to 2 or more antimicrobials. Prevalence of MRSA and ESBL E. coli and Klebsiella species isolates was also determined.

Univariate associations were determined between AMR profiles (susceptible or resistant) and source of isolates (IMI, subclinical mastitis, clinical mastitis) using chi-squared tests or Fisher's exact test (in case of expected cell frequencies $<5$ ). Data analyses were performed using Intercooled Stata 11.1 (Intercooled Stata for Macintosh, version 11.1, Stata Corp., Col- 
Table 2. Distribution of MIC of Escherichia coli $(\mathrm{n}=394)$ isolated from udders of dairy cattle on 76 dairy farms across 6 provinces in Canada $(2007-2008)^{1}$

\begin{tabular}{|c|c|c|c|c|c|c|c|c|c|c|c|c|c|c|c|c|c|c|}
\hline \multirow[b]{2}{*}{ Antimicrobial } & \multicolumn{16}{|c|}{$\mathrm{MIC}(\mu \mathrm{g} / \mathrm{mL})$} & \multirow[b]{2}{*}{$\mathrm{MIC}_{50}$} & \multirow[b]{2}{*}{$\mathrm{MIC}_{90}$} \\
\hline & $\leq 0.015$ & 0.03 & 0.06 & 0.12 & 0.25 & 0.5 & 1 & 2 & 4 & 8 & 16 & 32 & 64 & 128 & 256 & $>256$ & & \\
\hline Amikacin & & & & & & 0.5 & 15 & 67.6 & 14.7 & 1.6 & 0.3 & & & 0.3 & & & 2 & 4 \\
\hline Ampicillin & & & & & & & 15.5 & 47.3 & 27.0 & 0.5 & 1.5 & 0.7 & 7.5 & & & & 2 & 8 \\
\hline Amoxi-CLA $^{2}$ & & & & & & & 5.0 & 34.4 & 45.9 & 11.5 & 1.5 & 1.5 & 0.2 & & & & 4 & 8 \\
\hline Cefoxitin & & & & & & & 1.2 & 30.9 & 58.2 & 5.2 & 2.0 & 1.5 & 1.0 & & & & 4 & 4 \\
\hline Ceftriaxone & & & & & 98.0 & 0.2 & 0.5 & & 0.5 & & 0.8 & & & & & & $\leq 0.25$ & $\leq 0.25$ \\
\hline Ceftiofur & & & & 4.0 & 37.1 & 55.3 & 1.8 & 0.5 & 0.5 & 0.5 & 0.3 & & & & & & 0.5 & 0.5 \\
\hline Chloramphenicol & & & & & & & & 3.7 & 41.2 & 50.9 & 1.2 & & 3.0 & & & & 8 & 8 \\
\hline Gentamicin & & & & & 8.3 & 62.0 & 27.6 & 1.7 & & 0.2 & & 0.2 & & & & & 0.5 & 1 \\
\hline Kanamycin & & & & & & & & & & 94.3 & 1.0 & & 0.5 & 4.2 & & & $\leq 8$ & $\leq 8$ \\
\hline Streptomycin & & & & & & & & & & & & 91.2 & 3.5 & 5.2 & & & $\leq 32$ & $\leq 32$ \\
\hline Ciprofloxacin & 99.0 & 0.6 & 0.2 & & & & 0.2 & & & & & & & & & & $\leq 0.015$ & $\leq 0.015$ \\
\hline Nalidixic acid & & & & & & 0.2 & 10.5 & 75.1 & 14.0 & & & & 0.2 & & & & 2 & 2 \\
\hline Sulfisoxazole & & & & & & & & & & & 76.7 & 13.3 & 0.5 & 0.3 & & 9.2 & $\leq 16$ & 64 \\
\hline TMP-sulfa $^{3}$ & & & & 82.3 & 11.3 & 0.2 & 0.2 & 0.2 & & 5.8 & & & & & & & $\leq 0.12$ & 0.25 \\
\hline Tetracycline & & & & & & & & & 84.2 & 1.0 & & 5.8 & 9.0 & & & & $\leq 4$ & 32 \\
\hline \multicolumn{19}{|c|}{$\begin{array}{l}{ }^{1} \text { Numbers indicate percentage of isolates. Whi } \\
\text { greater than the highest concentration in the } \\
\text { those to the right being resistant. The } \mathrm{MIC}_{50} \\
{ }^{2} \text { Amoxicillin-clavulanic acid combination. } \\
{ }^{3} \text { Trimethoprim-sulfamethoxazole combination }\end{array}$} \\
\hline
\end{tabular}


lege Station, TX). In all instances, a $P$-value $<0.05$ was considered as statistically significant.

\section{RESULTS}

\section{Staphylococcus aureus}

Minimum inhibitory concentration values were determined for 562 isolates from 562 quarters of 462 cows on 79 dairy farms. Except for those of ceftiofur and sulfadimethoxine, the $\mathrm{MIC}_{90}$ values were less than or equal to the lowest antimicrobial concentration tested. Notably, the $\mathrm{MIC}_{90}$ value of sulfadimethoxine was 3 dilutions higher than its $\mathrm{MIC}_{50}$ value, whereas no difference in $\mathrm{MIC}_{50}$ and $\mathrm{MIC}_{90}$ value was observed for ceftiofur (Table 1). Resistance proportion estimates ranged from 0 for cephalothin and oxacillin to $8.8 \%$ for penicillin (Table 3). We observed no statistically significant differences in resistance proportion estimates of isolates from IMI and subclinical and clinical mastitis cases.

Among the 114 resistant Staph. aureus isolates, 17 (15.0\%) isolates were found to be MDR. Resistance to 2 antimicrobials was most common among MDR isolates. Ampicillin-penicillin, and penicillin-sulfadimethoxine resistance patterns were found in 9 and 1 isolates, respectively. One isolate exhibited an ampicillin-penicillin-sulfadimethoxine resistance pattern, whereas 2 isolates exhibited an ampicillin-penicillin-ceftiofur resistance pattern. Further, 2 isolates exhibited an erythromycin-penicillin-novobiocin combination-pirlimycin resistance pattern, whereas 1 isolate exhibited an erythromycin-penicillin-novobiocin combinationpirlimycin-sulfadimethoxine-tetracycline resistance pattern. Notably, 1 isolate also exhibited an erythromycin-pirlimycin resistance pattern (macrolide-lincosamide resistance).

In total, 1,802 Staph. aureus isolates were screened for methicillin resistance using chromogenic MRSA screening agar, and for penicillin resistance using Mueller-Hinton agar plates containing $0.25 \mu \mathrm{g} / \mathrm{mL}$ of penicillin $\mathrm{G}$, and 1 confirmed case of MRSA was identified (prevalence: $0.05 \%$ ). This isolate was not found among the original 562 isolates that were used for MIC testing, but among the additional 1,240 isolates that were screened for methicillin resistance. The MRSA isolate was susceptible to ceftiofur (MIC $=2 \mu \mathrm{g} / \mathrm{mL}$ ), cephalothin (MIC $\leq 2 \mu \mathrm{g} / \mathrm{mL}$ ), erythromycin (MIC $\leq 0.25 \mu \mathrm{g} /$ $\mathrm{mL}$ ), oxacillin ( $\mathrm{MIC} \leq 2 \mu \mathrm{g} / \mathrm{mL}$ ), penicillin-novobiocin combination ( $\mathrm{MIC} \leq 1 / 2 \mu \mathrm{g} / \mathrm{mL}$ ), and pirlimycin (MIC $\leq 0.5 \mu \mathrm{g} / \mathrm{mL}$ ), and resistant to ampicillin ( $\mathrm{MIC}=8 \mu \mathrm{g} /$ $\mathrm{mL}$ ), penicillin ( $\mathrm{MIC}=8 \mu \mathrm{g} / \mathrm{mL}$ ), sulfadimethoxine $(\mathrm{MIC}>256 \mu \mathrm{g} / \mathrm{mL}$ ), and tetracycline (MIC $>8 \mu \mathrm{g} /$ $\mathrm{mL}$ ). The MRSA isolate was confirmed for the presence of low-affinity penicillin binding protein PBP2'

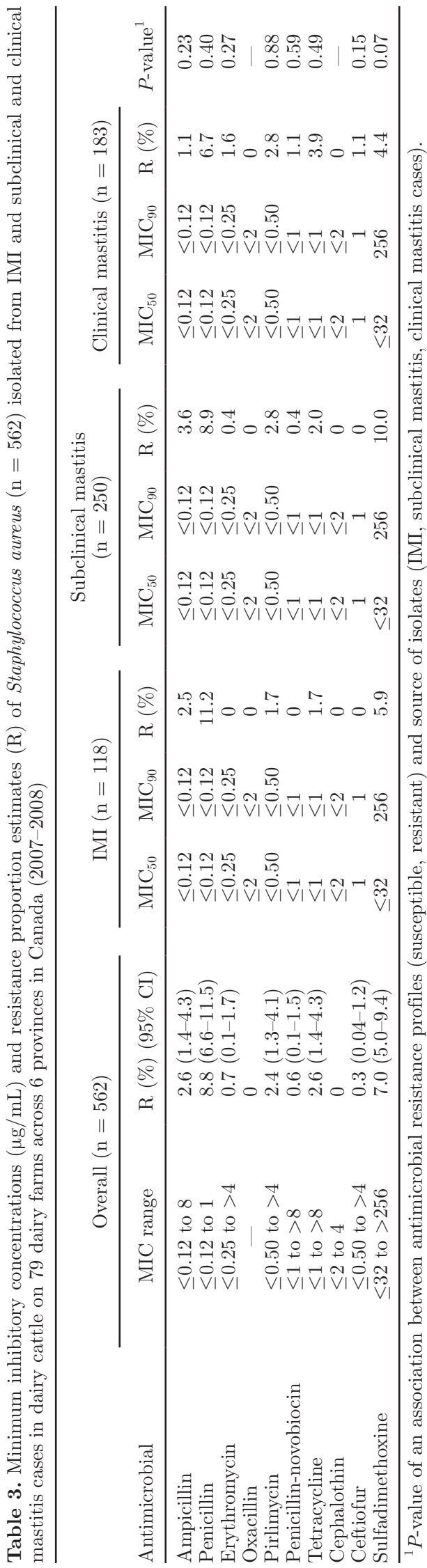

Journal of Dairy Science Vol. 95 No. 8, 2012 
and $m e c A$ gene. The prevalence of penicillin resistance determined using Mueller-Hinton agar plates containing $0.25 \mu \mathrm{g} / \mathrm{mL}$ of penicillin $\mathrm{G}$ was $6.9 \%(125 / 1,802$ isolates).

\section{Escherichia coli}

Minimum inhibitory concentration values were determined for 394 isolates from 394 quarters of 353 cows on 76 dairy farms. The majority of MIC values were below the resistance breakpoints. Notably, the $\mathrm{MIC}_{90}$ value of tetracycline was 3 dilutions higher than its $\mathrm{MIC}_{50}$ value (Table 2). Resistance proportions ranged from 0 for ceftriaxone and ciprofloxacin to $14.8 \%$ for tetracycline (Table 4). We found no difference in resistance proportions of isolates from IMI and subclinical and clinical mastitis cases and no difference in $\mathrm{MIC}_{50}$ and $\mathrm{MIC}_{90}$ values for the majority of antimicrobials. However, the $\mathrm{MIC}_{90}$ value of tetracycline in subclinical and clinical mastitis isolates was 3 dilutions higher than its $\mathrm{MIC}_{50}$ value. Further, the $\mathrm{MIC}_{90}$ value of sulfisoxazole in subclinical mastitis isolates was more than 4 dilutions higher than the $\mathrm{MIC}_{50}$ value, and $\mathrm{MIC}_{90}$ value of ampicillin in subclinical mastitis isolates was 3 dilutions higher than the $\mathrm{MIC}_{50}$ value. Five isolates were flagged as ESBL suspects due to reduced susceptibility to ceftriaxone (MIC $>2 \mu \mathrm{g} / \mathrm{mL}$ ). None was confirmed to be an ESBL producer based on the Sensititer ESBL confirm plate.

Of the 70 resistant $E$. coli isolates, $44(62.8 \%)$ isolates were found to be MDR (Table 5). Most commonly, 7 isolates were found resistant to 6 antimicrobials (ampicillin, kanamycin, streptomycin, sulfisoxazole, TMPsulfamethoxazole combination, and tetracycline). Notably, 1 isolate was found resistant to 10 antimicrobials (ampicillin, amoxicillin-CLA combination, chloramphenicol, ceftiofur, cefoxitin, kanamycin, streptomycin, sulfisoxazole, TMP-sulfamethoxazole combination, and tetracycline).

\section{Klebsiella Species}

Minimum inhibitory concentration values were determined for 139 isolates from 139 quarters of 114 cows on 37 dairy farms. The majority of MIC values were well below the resistance breakpoints. The $\mathrm{MIC}_{90}$ values of sulfisoxazole and tetracycline were 4 and 3 dilutions higher than $\mathrm{MIC}_{50}$ values, respectively (Table 6). Due to intrinsic resistance to ampicillin in Klebsiella species isolates, ampicillin resistance was not accounted for when determining the prevalence of AMR. Resistance proportions ranged from 0 for amikacin, ceftiofur, ceftriaxone, ciprofloxacin and nalidixic acid to $18.6 \%$ for tetracycline (Table 7). We found no differences in

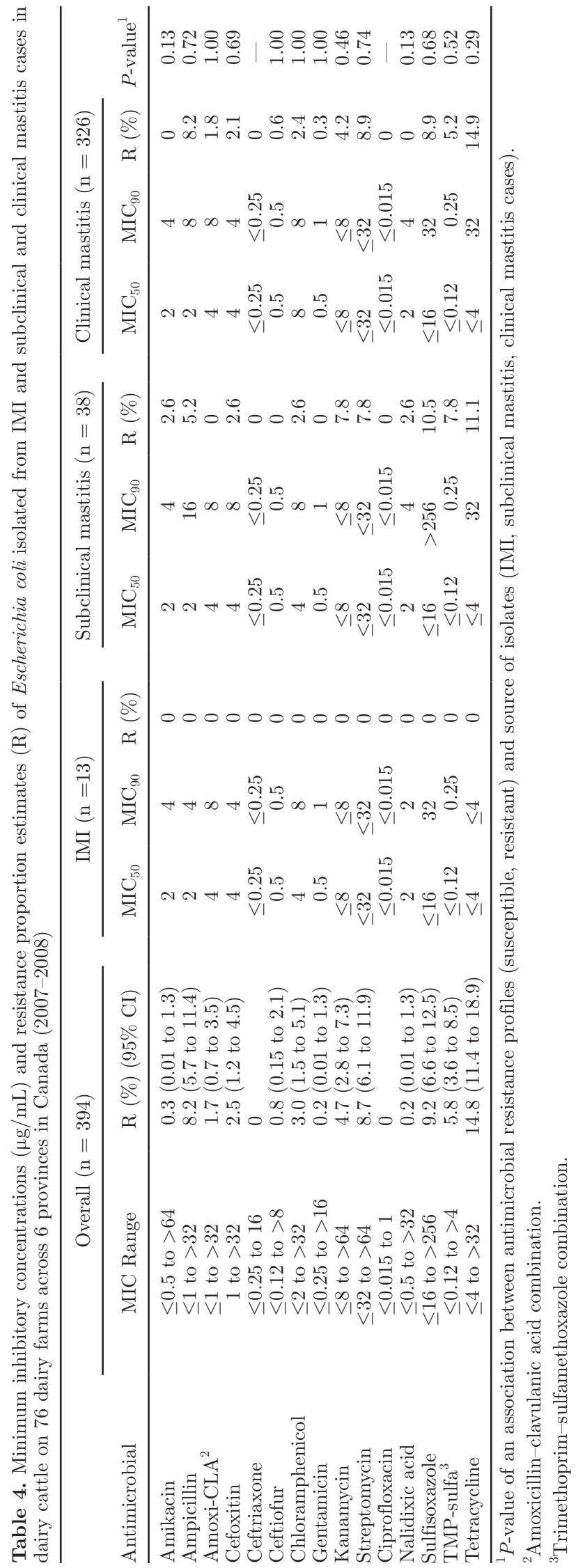

Journal of Dairy Science Vol. 95 No. 8, 2012 
Table 5. Multidrug resistance patterns in Escherichia coli $(\mathrm{n}=394)$ and Klebsiella species $(\mathrm{n}=139)$ isolated from udders of dairy cows across 6 provinces in Canada (2007-2008)

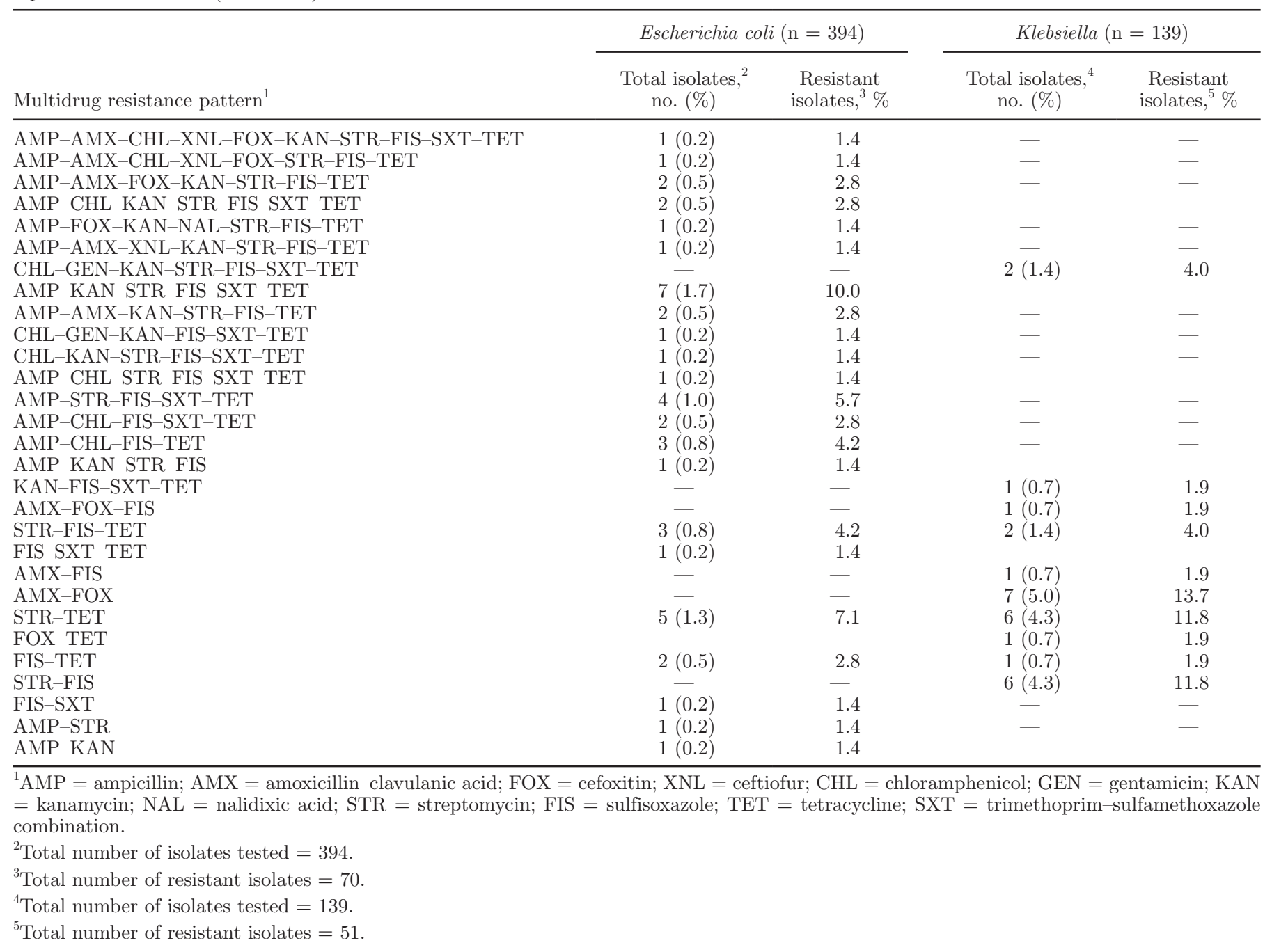

resistance proportions of isolates from IMI and subclinical and clinical mastitis cases. However, the $\mathrm{MIC}_{90}$ value of the amoxicillin-CLA combination in subclinical mastitis isolates was 4 dilutions higher than its $\mathrm{MIC}_{50}$ value. One isolate was flagged as ESBL suspect due to reduced susceptibility to ceftriaxone (MIC $>2 \mu \mathrm{g} / \mathrm{mL}$ ). However, it was not confirmed to be an ESBL producer based on Sensititer ESBL confirm plate.

Of the 51 resistant Klebsiella isolates, 28 (55.0\%) isolates were found to be MDR (Table 5). Two isolates were found resistant to 7 antimicrobials (chloramphenicol, gentamicin, kanamycin, streptomycin, sulfisoxazole, TMP-sulfamethoxazole combination, and tetracycline). Streptomycin-tetracycline, amoxicillin-CLA combination-cefoxitin, and streptomycin-sulfisoxazole resistance patterns were observed in 8,7 , and 6 isolates, respectively.

\section{DISCUSSION}

The study was designed to account for potential variation in AMR prevalence due to geographical and epidemiological differences. This is the first time that a Canada-wide study has been conducted to determine AMR in common mastitis pathogens, and prevalence of MRSA and ESBL E. coli and Klebsiella species isolated from milk samples in dairy cattle.

In general, more elaborate biochemical testing schemes and DNA-based diagnostic tests should be used for reliable identification of bovine mastitis pathogens for epidemiological research purposes. For example, some non-lactose-fermenting species of Enterobacteriaceae cannot be identified by biochemical testing schemes. Commercially produced miniaturized biochemical tests developed for identification of human clinical isolates 
Table 6. Distribution of MIC of Klebsiella species $(\mathrm{n}=139)$ isolated from udders of dairy cattle on 37 dairy farms across 6 provinces in Canada $(2007-2008)^{1}$

\begin{tabular}{|c|c|c|c|c|c|c|c|c|c|c|c|c|c|c|c|c|c|c|}
\hline \multirow[b]{2}{*}{ Antimicrobial } & \multicolumn{16}{|c|}{$\mathrm{MIC}(\mu \mathrm{g} / \mathrm{mL})$} & \multirow[b]{2}{*}{$\mathrm{MIC}_{50}$} & \multirow[b]{2}{*}{$\mathrm{MIC}_{90}$} \\
\hline & $\leq 0.015$ & 0.03 & 0.06 & 0.12 & 0.25 & 0.5 & 1 & 2 & 4 & 8 & 16 & 32 & 64 & 128 & 256 & $>256$ & & \\
\hline Amikacin & & & & & & 1.4 & 85.5 & 12.4 & 0.7 & & & & & & & & 1 & 2 \\
\hline Ampicillin & & & & & & & & & & & & 82.7 & 17.3 & & & & 32 & $>32$ \\
\hline Amoxi-CLA $^{2}$ & & & & & & & 13.1 & 66.9 & 9.7 & 3.4 & 1.4 & 2.1 & 3.4 & & & & 2 & 8 \\
\hline Cefoxitin & & & & & & & 6.2 & 65.5 & 19.3 & 1.4 & & 1.4 & 6.2 & & & & 2 & 4 \\
\hline Ceftriaxone & & & & & 98.6 & 0.7 & & & & 0.7 & & & & & & & $\leq 0.25$ & $\leq 0.25$ \\
\hline Ceftiofur & & & & 0.7 & 10.3 & 79.3 & 8.3 & 0.7 & 0.7 & & & & & & & & 0.5 & 0.5 \\
\hline Chloramphenicol & & & & & & & & 15.1 & 49.0 & 33.1 & 1.4 & & 1.4 & & & & 4 & 8 \\
\hline Gentamicin & & & & & 78.7 & 17.2 & 2.7 & & & & & 1.4 & & & & & $\leq 0.25$ & 0.5 \\
\hline Kanamycin & & & & & & & & & & 93.9 & & 2.0 & 2.8 & 1.3 & & & $\leq 8$ & $\leq 8$ \\
\hline Streptomycin & & & & & & & & & & & & 85.5 & 11.0 & 3.5 & & & $\leq 32$ & 64 \\
\hline Ciprofloxacin & 63.4 & 35.2 & 0.7 & 0.7 & & & & & & & & & & & & & $\leq 0.015$ & 0.03 \\
\hline Nalidixic acid & & & & & & & 4.1 & 86.2 & 9.7 & & & & & & & & 2 & 2 \\
\hline Sulfisoxazole & & & & & & & & & & & 60.0 & 20.7 & 4.8 & 2.8 & & 11.7 & $\leq 16$ & $>256$ \\
\hline TMP-sulfa $^{3}$ & & & & 67.6 & 29.7 & 0.7 & & & & 2.0 & & & & & & & $\leq 0.12$ & 0.25 \\
\hline Tetracycline & & & & & & & & & 80.7 & 0.7 & & 4.1 & 14.5 & & & & $\leq 4$ & $>32$ \\
\hline \multicolumn{19}{|c|}{$\begin{array}{l}{ }^{1} \text { Numbers indicate percentage of isolates. Whit } \\
\text { values greater than the highest concentration in } \\
\text { and those to the right being resistant. } \text { MIC }_{50} \text { an } \\
{ }^{2} \text { Amoxicillin-clavulanic acid combination. } \\
{ }^{3} \text { Trimethoprim-sulfamethoxazole combination. }\end{array}$} \\
\hline
\end{tabular}


are successful in delineating gram-negative bovine mastitis pathogens (Hogan and Smith, 2003). Diagnostic assays based on DNA that focus on the unique nucleic acid composition of the bacterial genome rather than expression of gene products have been used for reliable identification of common mastitis pathogens (Gillespie and Oliver, 2005).

The importance of accuracy and precision (i.e., validation of measuring instruments) in antimicrobial susceptibility testing cannot be understated. Antimicrobial susceptibility testing was done using Sensititer MIC system, which has recently been validated for bovine mastitis pathogens (Saini et al., 2011). Denim blue agar (chromogenic MRSA screening agar) was used for fast and accurate detection of methicillin resistance in Staph. aureus isolates; the agar contains cefoxitin that renders it selective to MRSA. The presence of low-affinity penicillin binding protein PBP2' in the MRSA isolate was confirmed using latex agglutination test. The PBP2' latex agglutination test has demonstrated high sensitivity $(96.9 \%)$ and specificity (100\%) in detecting MRSA (Yamazumi et al., 2001). Acquisition of PBP2' is encoded by the mecA gene. The MRSA isolate was positive on 2 real-time PCR assays for detecting femA gene (factor essential for methicillin resistance) and mecA gene in Staph. aureus (Paule et al., 2005). Interestingly, discrepancies between the MIC results and the results of denim blue chromogenic agar, latex agglutination test, and genotypic results were observed for this MRSA isolate; that is, this MRSA isolate was classified as susceptible according to CLSI breakpoint (oxacillin MIC $\leq 2 \mu \mathrm{g} / \mathrm{mL}$ ), and resistant due to presence of $\mathrm{PBP} 2^{\prime}$ and $m e c A$ gene as per denim blue chromogenic agar, latex agglutination test and genotypic results. It is probable that expression of methicillin-resistance in this MRSA strain was heterogeneous. Heterogeneous MRSA strains have apparently insufficient ability to synthesize PBP2' to provide a resistant phenotype, and some additional but yet to be identified factor(s) is needed for expression of methicillin-resistance in such strains (Hartman and Tomasz, 1986). Heterogeneous resistance expression is most difficult to detect using MIC determination methods because these methods classify such strains as susceptible (Cattoir and Leclercq, 2010). Therefore, methods used to detect mecA gene or the presence of PBP2' protein are considered reference methods for confirmation of methicillin-resistance in Staph. aureus isolates (CLSI, 2008; Cattoir and Leclercq, 2010).

Isolates were collected in a similar epidemiological manner on the basis of one isolate per quarter to ensure statistical independence. Multiple isolates of same species could be coming from the same quarter at different test days of sampling. Because this study 
was a cross-sectional snapshot of AMR situation, we randomly selected only one isolate per quarter so as not to contravene the statistical assumption of independence between isolates for AMR analysis. Collection of isolates from IMI and subclinical and clinical mastitis cases also helped to test the hypothesis that AMR proportion estimates differed among sources of isolates. However, such differences were not observed in the present study; a similar observation was made by Botrel et al. (2010). Multidrug resistance was defined as phenotypic resistance to 2 or more drugs either belonging to the same or different antimicrobial drug classes. For certain drug classes such as fluoroquinolones, resistance to different antimicrobials belonging to a drug class is mediated by a common resistance mechanism. In such cases, AMR is a class effect, and resistance to a class representative can be reasonably generalized to remaining antimicrobials in that drug class. However, in $\beta$-lactams and aminoglycosides, single class representatives cannot be defined due to the presence of potentially diverse resistance mechanisms. Antimicrobial resistance in such cases is not a class effect. Therefore, no universally accepted definition of MDR exists (Schwarz et al., 2010).

When comparing AMR proportion estimates and $\mathrm{MIC}_{90}$ values across studies, differences in antimicrobial susceptibility testing methods, interpretive criteria, source of isolates, sampling strategy, bacteriologic culturing and identification methods, and regional differences in pathogen populations should be borne in mind (Erskine et al., 2002). Most studies have determined MIC values or resistance proportion estimates in (sub) clinical Staph. aureus (n $=76$ to 2132), E. coli $(\mathrm{n}=93$ to 1939), and Klebsiella species isolates ( $\mathrm{n}=215$ to 637 ) using broth microdilution test method (Salmon et al., 1998; Sabour et al., 2004; Klement et al., 2005; FINRESVet, 2007; Srinivasan et al., 2007; MARAN-2008, 2009), agar dilution (Lehtolainen et al., 2003; Klement et al., 2005), agar disk diffusion test method (Gentilini et al., 2000; Erskine et al., 2002; Lanz et al., 2003; Makovec and Ruegg, 2003; Pengov and Ceru, 2003; Güler et al., 2005; Klement et al., 2005), or E-test (Pengov and Ceru, 2003; Güler et al., 2005). The majority of these studies used CLSI-based clinical breakpoints for AMR profiling. However, epidemiological cut-off values were used to categorize isolates in Finnish and Norwegian studies (FINRES-Vet, 2007; NORM/NORM-VET, 2009); the comparisons with these studies were made after adjusting for differences in breakpoints.

Among various antimicrobials tested, resistance to penicillin in Staph. aureus isolates was most commonly observed, as is reported in other studies (Erskine et al., 2002; Güler et al., 2005). However, $\mathrm{MIC}_{90}$ values and resistance proportion estimates for most antimicrobials were lower in our Canadian isolates than in most studies. For example, resistance proportion estimates for ampicillin, penicillin, erythromycin, and tetracycline ranged from 34.9 to $63.3 \%$ (Güler et al., 2005; Makovec and Ruegg, 2003), 9.9 to $63.3 \%$ (Güler et al., 2005; MARAN-2008, 2009), 0 to $11.6 \%$ (Gentilini et al., 2000; FINRES-Vet, 2007), and 8.6 to 27.9\% (Makovec and Ruegg, 2003; Güler et al., 2005), respectively, across studies, and they were higher than in our Canadian study $(2.6,8.8,0.7$, and $2.6 \%$, respectively). Resistance to oxacillin was absent, as also observed in many studies (Watts and Salmon, 1997; Pengov and Ceru, 2003; Güler et al., 2005; Klement et al., 2005). However, some studies observed higher resistance to oxacillin than was observed in our study: $0.6 \%$ (Erskine et al., 2002), 0.9\% (Sabour et al., 2004), 1.0\% (MARAN-2008, 2009), 1.8\% (Makovec and Ruegg, 2003), and 11.8\% (FINRES-Vet, 2007).

No Staph. aureus isolates were observed to be resistant to cephalothin and similar results were observed in various studies (Watts and Salmon, 1997; Sabour et al., 2004; Klement et al., 2005; FINRES-Vet, 2007). Cephalothin is used as a class representative of firstgeneration cephalosporins except cefazolin in antimicrobial susceptibility testing (CLSI, 2008). Cephapirin, a first-generation cephalosporin, is commonly used for dry cow therapy and intramammary clinical mastitis treatment, whereas ceftiofur hydrochloride, a third-generation cephalosporin, is administered intramammary for clinical mastitis treatment. Cephalothin resistance $(0 \%)$ in our Canadian isolates was lower than in many other studies $(0.1,0.2,1.0 \%$, respectively, in Erskine et al., 2002; Makovec and Ruegg, 2003; MARAN-2008, 2009). Ceftiofur resistance $(0.3 \%)$ in our study was similar to that in the US studies $(0.1 \%, 0.2 \%$; Erskine et al., 2002; Makovec and Ruegg, 2003) and higher than in Canadian and New Zealand studies, in which no resistant isolates were observed (Salmon et al., 1998; Sabour et al., 2004). Generally, resistance to $\beta$-lactams (penicillins and cephalosporins) was low in bovine mastitis Staph. aureus isolates, despite the fact that $\beta$-lactams are most commonly used for mastitis treatment and control in dairy cattle (Saini et al., 2012). Commonly employed "full-dose short-term" treatment regimens are unlikely to promote dissemination of resistance determinants in an udder environment (WHO, 1997) and could explain the lower prevalence of AMR in bovine mastitis pathogens. Resistance to pirlimycin and the penicillin-novobiocin combination was rare, as also reported in various studies (Gentilini et al., 2000; Makovec and Ruegg, 2003; MARAN-2008, 2009). Pirlimycin, a lincosamide antimicrobial, is indicated for intramammary treatment of clinical and subclinical mastitis in lactating dairy cattle, whereas 
the penicillin-novobiocin combination is indicated for intramammary treatment and prevention of mastitis in dry cows. In general, the study results suggest that most intramammary antimicrobials are still effective for treatment and control of bovine mastitis despite being extensively used.

In the case of $E$. coli, resistance to tetracyclines was most common followed by sulfonamides, ampicillin, TMP-sulfonamide combination, and aminoglycosides in declining order. Widespread acquired resistance to tetracyclines could be due to extensive use over many years. Sulfonamides are also occasionally used to treat septicemia caused by coliform mastitis in dairy cattle (Erskine et al., 2002). Tetracycline (14.8\%) and sulfisoxazole $(9.2 \%)$ resistance proportion estimates were lower in our Canadian E. coli isolates than in most studies. For example, published resistance proportion estimates of tetracycline and sulfisoxazole range from 16.2 to $37.4 \%$ (Makovec and Ruegg, 2003; MARAN-2008, 2009 ) and 16.3 to $34.1 \%$ (Makovec and Ruegg, 2003; Srinivasan et al., 2007), respectively, across studies. Resistance to TMP-sulfamethoxazole combination was low $(5.8 \%)$, potentially due to synergistic bactericidal activity of TMP and sulfonamides in a combination.

In coliform mastitis cases with signs of systemic illness, ceftiofur, ampicillin, and amoxicillin can be used for systemic treatment in lactating dairy cattle (Wagner and Erskine, 2006). Fewer E. coli isolates were resistant to ampicillin (8.2\%) than in most studies (9.7 to $98.4 \%$; Srinivasan et al., 2007; Botrel et al., 2010). Cephalosporins such as cefoxitin, ceftriaxone, and ceftiofur have high antibacterial activity against most Enterobacteriaceae and are broadly resistant to $\beta$-lactamases. Resistance to ceftiofur (0.8\%) and cefoxitin (2.5\%) was uncommon and ceftriaxone was absent in our Canadian isolates. Ceftiofur resistance proportion estimate $(0.8 \%)$ and $\mathrm{MIC}_{90}$ value $(0.5 \mu \mathrm{g} / \mathrm{mL})$ in E. coli isolates in our study were similar to those in the Finnish and Norwegian studies $(0 \%, 0.5 \mu \mathrm{g} / \mathrm{mL}$; FINRES-Vet, 2007; NORM/NORM-VET, 2009). However, ceftiofur resistance proportion estimate in E. coli $(0.8 \%)$ and Klebsiella (0\%) species isolates was far lower than in a US study by Erskine et al. (2002), in which $4.6 \%$ of E. coli and $14.1 \%$ of Klebsiella species isolates were resistant. Further, reduced susceptibility to ceftriaxone (MIC $\geq 2 \mu \mathrm{g} / \mathrm{mL}$ ) is an indicator of ESBL production in Enterobacteriaceae. However, no ESBL-producing $E$. coli isolates were observed, as reported in other studies (FINRES-Vet, 2007; NORM/NORM-VET, 2009). Interestingly, the $\mathrm{MIC}_{90}$ value of ceftiofur was higher than that of ceftriaxone in coliforms (0.5 vs. $\leq 0.25 \mu \mathrm{g} /$ $\mathrm{mL}$, respectively). Ceftriaxone is not indicated for use in dairy cattle, whereas ceftiofur is extensively used (Saini et al., 2012). However, ceftiofur is poorly distrib- uted in the udder due to high plasma protein binding upon systemic administration (Prescott, 2006). Therefore, it is likely that subtherapeutic concentrations of ceftiofur achieved in the udder may cause selection of ceftiofur resistance in coliforms. In general, resistance to antimicrobials of very high importance to human medicine (as categorized by Health Canada, 2009) such as third-generation cephalosporins (ceftiofur, ceftriaxone), penicillin- $\beta$-lactamase inhibitor combinations (amoxicillin-CLA combination), and fluoroquinolones (ciprofloxacin) is uncommon to rare in bovine mastitis coliforms. The study results suggest a low risk of transmission of AMR bacteria from milk or milk products to human populations.

Multidrug resistance was frequently observed in resistant gram-negative bovine mastitis pathogens. The majority of MDR E. coli were resistant to kanamycin, streptomycin, ampicillin, sulfisoxazole, TMP-sulfamethoxazole combination, and tetracycline. Linkage between resistance determinants of sulfonamides (sul1 and sul2 genes) and TMP ( $d f r 1$ gene) or streptomycin (aadA1a) is very common; co-location of resistance determinants on specific plasmids (thereby leading to linkage of resistance genes) could explain the large number of MDR patterns in E. coli isolates (Lanz et al., 2003). Multidrug resistance to ampicillin, streptomycin, sulfisoxazole, and tetracycline was also very commonly observed in E. coli isolated from bovine mastitis cases in the study by Srinivasan et al. (2007). However, the proportion of MDR isolates was far higher in their study (90.7\%). Lehtolainen et al. (2003) also reported that tetracycline-dihydrostreptomycin-ampicillin was the most frequent AMR pattern in MDR E. coli isolated from acute clinical bovine mastitis cases in Israel and Finland in their study. In general, the occurrence of multiple resistance in gram-negative bovine mastitis pathogens needs to be monitored in the coming years as also advised by Dutch and Norwegian AMR surveillance programs (MARAN-2008, 2009; NORM/NORMVET, 2009).

\section{CONCLUSIONS}

In general, AMR prevalence was uncommon in the bovine mastitis Staph. aureus, E. coli, and Klebsiella species pathogens. Differences in resistance proportion estimates among isolates from IMI and subclinical and clinical mastitis cases were not observed. Multidrug resistance was more commonly observed in E. coli and Klebsiella species than in Staph. aureus isolates. Resistance to antimicrobials of very high importance in human medicine was rare in bovine mastitis pathogens. Only 1 out of 1,802 Staph. aureus isolates screened was positive for MRSA (prevalence of $0.05 \%$ ), and no ESBL 
E. coli and Klebsiella species isolates were found in our Canadian milk samples. The study results suggest a low risk of transmission of AMR bacteria from milk or milk products to human populations.

\section{ACKNOWLEDGMENTS}

The authors thank technicians Adele Veinot, Andrea Wasko, Anke Wellen, Francois Dubois, Meliza Morris, Mike MacLean, Natasha Robinson and Theresa Andrews for collection of milk samples, Matthew Saab and Cynthia Mitchell for all laboratory analyses (all from Canadian Bovine Mastitis Research Network, StHyacinthe, QC, Canada), Vicky Stagg (University of Calgary, AB, Canada) for statistical programming, and Trevor DeVries, Jean-Philippe Roy, Luc DesCôteaux, Ian Dohoo, and Kristen Reyher (all from Canadian Bovine Mastitis Research Network, St-Hyacinthe, QC, Canada) for coordination of the National Dairy Farm Cohort regional centres. This research was financed by the Natural Science and Engineering Research Council (Ottawa, ON, Canada), Alberta Milk (Edmonton, AB, Canada), Dairy Farmers of New Brunswick, Nova Scotia, Ontario and Prince Edward Island, Novalait Inc. (Québec, QC, Canada), Dairy Farmers of Canada (Ottawa, ON, Canada), Canadian Dairy Network (Guelph, ON, Canada), Agriculture and Agri-Food Canada(Ottawa, ON, Canada), Public Health Agency of Canada (Ottawa, ON, Canada), Technology PEI Inc. (Charlottetown, PEI, Canada), Université de Montréal (Montréal, QC, Canada) and University of Prince Edward Island (Charlottetown, PEI, Canada), through the Canadian Bovine Mastitis Research Network.

\section{REFERENCES}

Barkema, H. W., Y. H. Schukken, T. J. G. M. Lam, M. L. Beiboer, H. Wilmink, G. Benedictus, and A. Brand. 1998. Incidence of clinical mastitis in dairy herds grouped in three categories by bulk milk somatic cell counts. J. Dairy Sci. 81:411-419.

Barkema, H. W., Y. H. Schukken, and R. N. Zadoks. 2006. Invited review: The role of cow, pathogen, and treatment regimen in the therapeutic success of bovine Staphylococcus aureus mastitis. J. Dairy Sci. 89:1877-1895.

Berry, D. P., and W. J. Meaney. 2006. Interdependence and distribution of subclinical mastitis and intramammary infection among udder quarters in dairy cattle. Prev. Vet. Med. 75:81-91.

Botrel, M.-A., M. Haenni, E. Morignat, P. Sulpice, J.-Y. Madec, and D. Calavas. 2010. Distribution and antimicrobial resistance of clinical and subclinical mastitis pathogens in dairy cows in RhôneAlpes, France. Foodborne Pathog. Dis. 7:479-487.

Cattoir, V., and R. Leclercq. 2010. $\beta$-Lactams and staphylococci. Pages 102-105 in Antibiogram. P. Courvalin, R. Leclercq, L. B. Rice, eds. ESKA Publishing, Portland, OR.

CLSI. 2000a. Methods for dilution antimicrobial susceptibility tests for bacteria that grow aerobically. Approved Standard M7-A5. 5th ed. Clinical and Laboratory Standards Institute, Wayne, PA.

CLSI. 2000b. Performance standards for antimicrobial susceptibility testing. 12th Informational Supplement. M100-S14. Clinical and Laboratory Standards Institute, Wayne, PA.
CLSI. 2008. Performance Standards for Antimicrobial Disk and Dilution Susceptibility Tests for Bacteria Isolated From Animals. Pages 65-72 in Approved Standard M31-A3. 3rd ed. Clinical and Laboratory Standards Institute, Wayne, PA.

Dohoo, I. R., J. Smith, S. Andersen, D. F. Kelton, and S. Godden., and Mastitis Research Workers' Conference. 2011. Diagnosing intramammary infections: Evaluation of definitions based on a single milk sample. J. Dairy Sci. 94:250-261.

Dolejska, M., Z. Jurcickova, I. Literak, L. Pokludova, J. Bures, A Hera, L. Kohoutova, J. Smola, and A. Cizek. 2011. IncN plasmids carrying bla $a_{\mathrm{CTX}-\mathrm{M}-1}$ in Escherichia coli isolates on a dairy farm. Vet. Microbiol. 149:513-516.

Erskine, R. J., R. D. Walker, C. A. Bolin, P. C. Bartlett, and D. G. White. 2002. Trends in antibacterial susceptibility of mastitis pathogens during a seven-year period. J. Dairy Sci. 85:1111-1118.

Fey, P. D., T. J. Safranek, M. E. Rupp, E. F. Dunne, E. Ribot, P. C. Iwen, P. A. Bradford, F. J. Angulo, and S. H. Hinrichs. 2000. Ceftriaxone-resistant salmonella infection acquired by a child from cattle. N. Engl. J. Med. 342:1242-1249.

FINRES-Vet. 2007. Finnish veterinary antimicrobial resistance monitoring and consumption of antimicrobial agents. Finnish Food Safety Authority Evira, Helsinki, Finland. Accessed Jan. 23, 2012. http://www.evira.fi/portal/en/evira/publications/?a=view\&prod uctId $=178$.

Gentilini, E., G. Denamiel, P. Llorente, S. Godaly, M. Rebuelto, and O. DeGregorio. 2000. Antimicrobial susceptibility of Staphylococcus aureus isolated from bovine mastitis in Argentina. J. Dairy Sci. 83:1224-1227.

Gillespie, B. E., and S. P. Oliver. 2005. Simultaneous detection of mastitis pathogens, Staphylococcus aureus, Streptococcus uberis, and Streptococcus agalactiae by multiplex real-time polymerase chain reaction. J. Dairy Sci. 88:3510-3518.

Güler, L., U. Ok, K. Gündüz, Y. Gülcü, and H. H. Hadimli. 2005. Antimicrobial susceptibility and coagulase gene typing of Staphylococcus aureus isolated from bovine clinical mastitis cases in Turkey. J. Dairy Sci. 88:3149-3154.

Hammad, A. M., A. M. Ahmed, Y. Ishida, and T. Shimamoto. 2008. First characterization and emergence of SHV-60 in raw milk of a healthy cow in Japan. J. Vet. Med. Sci. 70:1269-1272.

Hariharan, H., D. A. Barnum, and W. R. Mitchell. 1974. Drug resistance among pathogenic bacteria from animals in Ontario. Can. J. Comp. Med. 38:213-221.

Hartman, B. J., and A. Tomasz. 1986. Expression of methicillin-resistance in heterogeneous strains of Staphylococcus aureus. Antimicrob. Agents Chemother. 29:85-92.

Health Canada. 2009. Categorization of antimicrobial drugs based on importance in human medicine. Accessed Jan. 23, 2012. http:// www.hc-sc.gc.ca/dhp-mps/consultation/vet/consultations/amr_ ram_hum-med-rev-eng.php.

Hogan, J., and K. L. Smith. 2003. Coliform mastitis. Vet. Res. 34:507-519.

Hogan, J. S., R. N. Gonzalez, R. Harmon, S. C. Nickerson, S. P. Oliver, J. W. Pankey, and K. L. Smith. 1999. Laboratory Handbook on Bovine Mastitis. National Mastitis Council Inc., Verona, WI.

Klement, E., M. Chaffer, G. Leitner, A. Shwimmer, S. Friedman, A. Saran, and N. Shpigel. 2005. Assessment of accuracy of disk diffusion tests for the determination of antimicrobial susceptibility of common bovine mastitis pathogens: A novel approach. Microb. Drug Resist. 11:342-350.

Lanz, R., P. Kuhnert, and P. Boerlin. 2003. Antimicrobial resistance and resistance gene determinants in clinical Escherichia coli from different animal species in Switzerland. Vet. Microbiol. 91:73-84.

Lehtolainen, T., A. Shwimmer, N. Y. Shpigel, T. Honkanen-Buzalski, and S. Pyörälä. 2003. In vitro antimicrobial susceptibility of Escherichia coli isolates from clinical bovine mastitis in Finland and Israel. J. Dairy Sci. 86:3927-3932.

Macdonald, K., J. Greenfield, and C. Andress. 1973. Current antibiograms for selected bacterial pathogens isolated from animals and poultry in British Columbia. Can. Vet. J. 14:291-292.

Makovec, J. A., and P. L. Ruegg. 2003. Antimicrobial resistance of bacteria isolated from dairy cow milk samples submitted for bacte- 
rial culture: 8,905 samples (1994-2001). J. Am. Vet. Med. Assoc. $222: 1582-1589$.

MARAN-2008. 2009. MARAN-2008-Monitoring of antimicrobial resistance and antibiotic usage in animals in the Netherlands in 2008. Accessed Jan. 23, 2012. http://www.cvi.wur.nl/NR/rdonlyres/DDA15856-1179-4CAB-BAC6-28C4728ACA03/110563/ MARAN_2008__definitief_corrected.pdf.

NARMS (National Antimicrobial Resistance Monitoring System). 2011. United States Food and Drug Administration. Department of Health and Human Services. Accessed Jan. 23, 2012. http:// www.fda.gov/AnimalVeterinary/SafetyHealth/AntimicrobialResistance/NationalAntimicrobialResistanceMonitoringSystem/default.htm.

NCDF (National Cohort of Dairy Farms). 2009. National Cohort of Dairy Farms Reference Manual. Accessed Jan. 23, 2012. http:// www.mastitisnetwork.org.

NORM/NORM-VET. 2009. Usage of antimicrobial agents and occurrence of antimicrobial resistance in Norway. Troms $\varnothing /$ Oslo, Norway. Accessed Jan. 23, 2012. http://www.vetinst.no/eng/ Research/Publications/Norm-Norm-Vet-Report/Norm-Norm-Vetreport-2009.

Oliver, S. P., B. M. Jayarao, and R. A. Almeida. 2005. Foodborne pathogens in milk and the dairy farm environment: Food safety and public health implications. Foodborne Pathog. Dis. 2:115129.

Paule, S. M., A. C. Pasquariello, R. B. Thomson, K. L. Kaul, and L. R. Peterson. 2005. Real-time PCR can rapidly detect methicillin-susceptible and methicillin-resistant Staphylococcus aureus directly from positive blood culture bottles. Am. J. Clin. Pathol. 124:404-407.

Pengov, A., and S. Ceru. 2003. Antimicrobial drug susceptibility of Staphylococcus aureus strains isolated from bovine and ovine mammary glands. J. Dairy Sci. 86:3157-3163.

Piddock, L. J. 1996. Does the use of antimicrobial agents in veterinary medicine and animal husbandry select antibiotic-resistant bacteria that infect man and compromise antimicrobial chemotherapy? J. Antimicrob. Chemother. 38:1-3.

Prescott, F. J. 2006. Beta-lactam antibiotics: Cephalosporins. Page 151 in Antimicrobial Therapy in Veterinary Medicine. S. Giguère, ed. Blackwell Publishing, Ames, IA.

Reyher, K. K., S. Dufour, H. W. Barkema, L. Des Côteaux, T. J. Devries, I. R. Dohoo, G. P. Keefe, J.-P. Roy, and D. T. Scholl. 2011. The National Cohort of Dairy Farms - A data collection platform for mastitis research in Canada. J. Dairy Sci. 94:16161626.

Sabour, P. M., J. J. Gill, D. Lepp, J. C. Pacan, R. Ahmed, R. Dingwell, and K. Leslie. 2004. Molecular typing and distribution of Staphylococcus aureus isolates in Eastern Canadian dairy herds. J. Clin. Microbiol. 42:3449-3455.
Saini, V., J. T. McClure, D. Léger, S. Dufour, A. G. Sheldon, D. T Scholl, and H. W. Barkema. 2012. Antimicrobial use on Canadian dairy farms. J. Dairy Sci. 95:1209-1221.

Saini, V., R. G. M. Olde Riekerink, J. T. McClure, and H. W. Barkema. 2011. Diagnostic accuracy assessment of Sensititre and agar disk diffusion for determining antimicrobial resistance profiles of bovine clinical mastitis pathogens. J. Clin. Microbiol. 49:15681577 .

Salmon, S. A., J. L. Watts, F. M. Aarestrup, J. W. Pankey, and R. J. Yancey. 1998. Minimum inhibitory concentrations for selected antimicrobial agents against organisms isolated from the mammary glands of dairy heifers in New Zealand and Denmark. J. Dairy Sci. 81:570-578.

Schwarz, S., P. Silley, S. Simjee, N. Woodford, E. van Duijkeren, A. P. Johnson, and W. Gaastra. 2010. Editorial: Assessing the antimicrobial susceptibility of bacteria obtained from animals. J. Antimicrob. Chemother. 65:601-604.

Srinivasan, V., B. E. Gillespie, M. J. Lewis, L. T. Nguyen, S. I. Headrick, Y. H. Schukken, and S. P. Oliver. 2007. Phenotypic and genotypic antimicrobial resistance patterns of Escherichia coli isolated from dairy cows with mastitis. Vet. Microbiol. 124:319-328.

Vanderhaeghen, W., T. Cerpentier, C. Adriaensen, J. Vicca, K. Hermans, and P. Butaye. 2010. Methicillin-resistant Staphylococcus aureus (MRSA) ST398 associated with clinical and subclinical mastitis in Belgian cows. Vet. Microbiol. 144:166-171.

Wagner, S., and R. Erskine. 2006. Antimicrobial drug use in bovine mastitis. Page 511 in Antimicrobial Therapy in Veterinary Medicine. S. Giguère, J. F. Prescott, J. D. Baggot, R. D. Walker, and P. M. Dowling, ed. Iowa State University Press, Ames.

Watts, J. L., and S. A. Salmon. 1997. Activity of selected antimicrobial agents against strains of Staphylococcus aureus isolated from bovine intramammary infections that produce beta-lactamase. J. Dairy Sci. 80:788-791.

World Health Organization (WHO). 1997. Pages 11-16 in Recommendations in WHO Proceedings: The Medical Impact of Antimicrobial use in Food Animals. WHO/EMC/ZOO/97.4, 1997. WHO, Geneva, Switzerland.

Yamazumi, T., S. A. Marshall, W. W. Wilke, D. J. Diekema, M. A. Pfaller, and R. N. Jones. 2001. Comparison of the Vitek grampositive susceptibility 106 card and the MRSA-screen latex agglutination test for determining oxacillin resistance in clinical bloodstream isolates of Staphylococcus aureus. J. Clin. Microbiol. 39:53-56.

Zadoks, R. N., and M. A. Muñoz. 2007. The emergence of Klebsiella as a major mastitis organism. Pages $100-111$ in Proc. Natl. Mastitis Counc. 46th Annu. Mtg., San Antonio, TX. National Mastitis Council Inc., Verona, WI. 